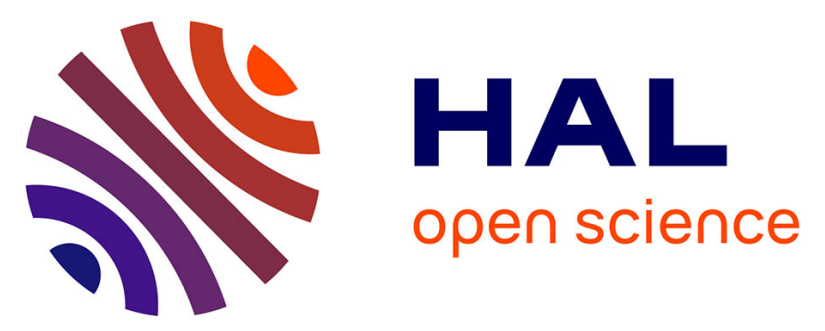

\title{
Dephasing effects on coherent exciton-polaritons and the breakdown of the strong coupling regime
}

Naotomo Takemura, Mitchell D. Anderson, Stephane Trebaol, Souvik Biswas, Daniel Oberli, Marcia T. Portella-Oberli, Benoit Deveaud

\section{- To cite this version:}

Naotomo Takemura, Mitchell D. Anderson, Stephane Trebaol, Souvik Biswas, Daniel Oberli, et al.. Dephasing effects on coherent exciton-polaritons and the breakdown of the strong coupling regime. Physical Review B: Condensed Matter and Materials Physics (1998-2015), 2015, 92 (23), pp.235305. 10.1103/PhysRevB.92.235305 . hal-01254979

\section{HAL Id: hal-01254979 \\ https://hal.science/hal-01254979}

Submitted on 13 Jan 2016

HAL is a multi-disciplinary open access archive for the deposit and dissemination of scientific research documents, whether they are published or not. The documents may come from teaching and research institutions in France or abroad, or from public or private research centers.
L'archive ouverte pluridisciplinaire HAL, est destinée au dépôt et à la diffusion de documents scientifiques de niveau recherche, publiés ou non, émanant des établissements d'enseignement et de recherche français ou étrangers, des laboratoires publics ou privés. 


\title{
Dephasing effects on coherent exciton-polaritons and the breakdown of the strong coupling regime
}

\author{
N. Takemura, ${ }^{1, *}$ M. D. Anderson, ${ }^{1}$ S. Trebaol, ${ }^{2}$ S. Biswas, ${ }^{1}$ D. Y. Oberli, ${ }^{1}$ M. T. Portella-Oberli, ${ }^{1}$ and B. Deveaud ${ }^{1}$ \\ ${ }^{1}$ Laboratory of Quantum Optoelectronics, École Polytechnique Fédérale de Lausanne, CH-1015, Lausanne, Switzerland \\ ${ }^{2}$ UMR FOTON, CNRS, Université de Rennes 1, Enssat, F22305 Lannion, France
}

(Received 30 July 2015; revised manuscript received 18 November 2015; published 15 December 2015)

\begin{abstract}
Using femtosecond pump-probe spectroscopy, we identify excitation-induced dephasing as a major mechanism responsible for the breakdown of the strong coupling between excitons and photons in a semiconductor microcavity. The effects of dephasing are observed on the transmitted probe-pulse spectrum as a density-dependent broadening of the exciton-polariton resonances and the emergence of a third resonance at high excitation density. A striking asymmetry in the energy shift between the upper and the lower polaritons is also evidenced. Using the excitonic Bloch equations, we quantify the respective contributions to the energy shift of many-body effects associated with Coulomb fermion exchange and photon assisted exchange processes and the contribution to collisional broadening.
\end{abstract}

DOI: 10.1103/PhysRevB.92.235305

PACS number(s): 71.36. $+\mathrm{c}, 42.65 .-\mathrm{k}, 76.50 .+\mathrm{g}, 78.20 . \mathrm{Ls}$

\section{INTRODUCTION}

A semiconductor microcavity is a system that confines photons and allows them to strongly interact with quantum well excitons [1]. Polaritons are composite particles arising from the coherent superposition of a photon and an exciton. The polariton interactions, mediated by their excitonic part, make semiconductor microcavities a suitable platform for investigating a wide range of physical phenomena where coherence is a major issue, including Bose-Einstein condensation [2,3] or collective quantum fluid effects [4-6]. Experimentally, the coherent dynamics of polaritons have been investigated using four-wave mixing [5-9] and pumpprobe techniques [10-13], where the excitonic nonlinearity is evidenced through the changes in the optical response of the system. Theoretically, the coherent dynamics of polaritons are usually modeled using a nonlinear Schrödinger equation, which is formally equivalent to a Gross-Pitaevskii equations (GPE), often used for describing the coherent ground state of Bose condensed dilute atoms.

On the other hand, the exciton-exciton interactions play an important role in the coherent properties of semiconductor systems because of the dephasing [14-16]. The investigation of the effect of exciton-exciton interactions on the polariton dynamics is important both for understanding the fundamental physics of coherent exciton-photon strongly coupled systems and for designing semiconductor microcavity devices. For instance, the presence of an exciton reservoir interacting with polaritons plays a major role in the spontaneous formation of polariton condensation following nonresonant excitation [17], as well as in the behavior of the polariton multistability [18]. However, the effect of excitation induced dephasing in the context of the coherent polariton dynamics has yet to be considered. We show here that, after the resonantly coherent excitation of both the lower and upper polaritons, the exciton-exciton interaction converts the coherent polariton population into an incoherent exciton population. This excitation induced dephasing process affects the polariton dynamics and is a major mechanism in the breakdown of the exciton-photon strong coupling.

*naotomo.takemura@epfl.ch
In this paper, we show that excitation-induced dephasing (EID) plays an important role in the dynamics of polaritons in a semiconductor microcavity. The investigation is experimentally carried out by femtosecond pump-probe optical spectroscopy. For the theoretical description of our results, we utilize the excitonic Bloch equations (EBE) approach, taking into account separately the coherent part of the polariton population and an incoherent population of excitons [19,20]. We study the role of exciton-exciton interactions, photonassisted exchange scattering, and EID effects on the polariton dynamics. The experimental results are very well reproduced by EBE and not by the exciton-photon GPE, which assumes that excitons stay in the coherent limit.

\section{EXPERIMENT}

The experiment is performed with a high quality GaAsbased microcavity [21] at the cryogenic temperature of $4 \mathrm{~K}$. A single $8 \mathrm{~nm} \operatorname{In}_{0.04} \mathrm{Ga}_{0.96}$ As quantum well is embedded between two GaAs/AlAs distributed Bragg reflectors. The Rabi splitting energy is $2 \Omega=3.45 \mathrm{meV}$ at zero cavity detuning [5]. For the accurate measurement of the transmitted probe beam, we employ a heterodyne pump-probe setup [12] with a degenerate beam configuration at $\boldsymbol{k}=0 \mu \mathrm{m}^{-1}$, which dramatically increases the signal-to-noise ratio. The pump and probe pulses originate both from a broadband few hundreds femtosecond $\mathrm{Ti}$ : Sapphire laser. The center of the laser spectrum is set between the lower- and upper-polariton peaks. Additionally, noise coming from laser spectrum envelope is removed with the aid of a numerical low-pass filter. The experimental setup is described in detail in our previous papers $[6,12]$. In order to avoid the complex effects of biexcitons $[12,13]$, the pump and probe beams are cocircularly polarized. We obtain a time delay map in frequency and in real time through successive measurements of the pump-probe spectrum.

Typical experimental results are presented in Figs. 1(a) and $1(\mathrm{c})$, showing the probe spectra as a function of pumpprobe time delay. The cavity detuning is set at $\epsilon_{c}-\epsilon_{x}=$ $0.8 \mathrm{meV}$, where $\epsilon_{c(x)}$ is cavity mode (exciton) energy. In this figure, the pump pulse arrives before (after) the probe pulse at positive (negative) pump-probe delays. For low pump intensity [Fig. 1(a)], we observe two polariton branches (lower and 

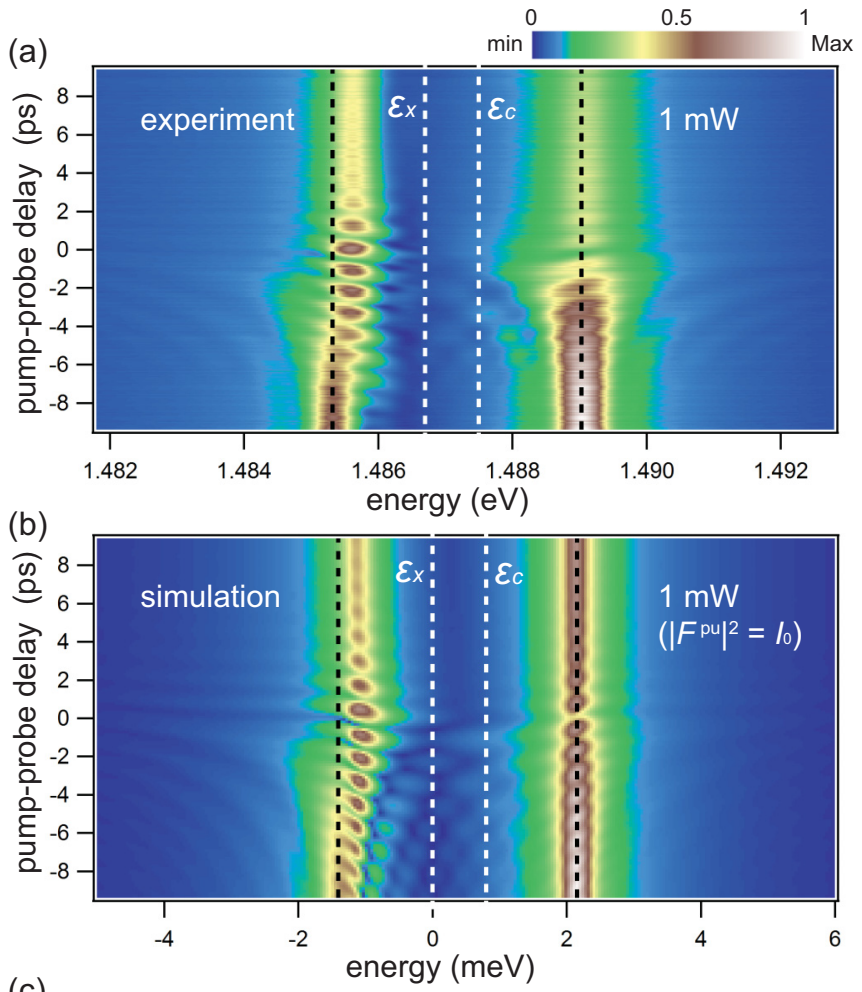

(c)
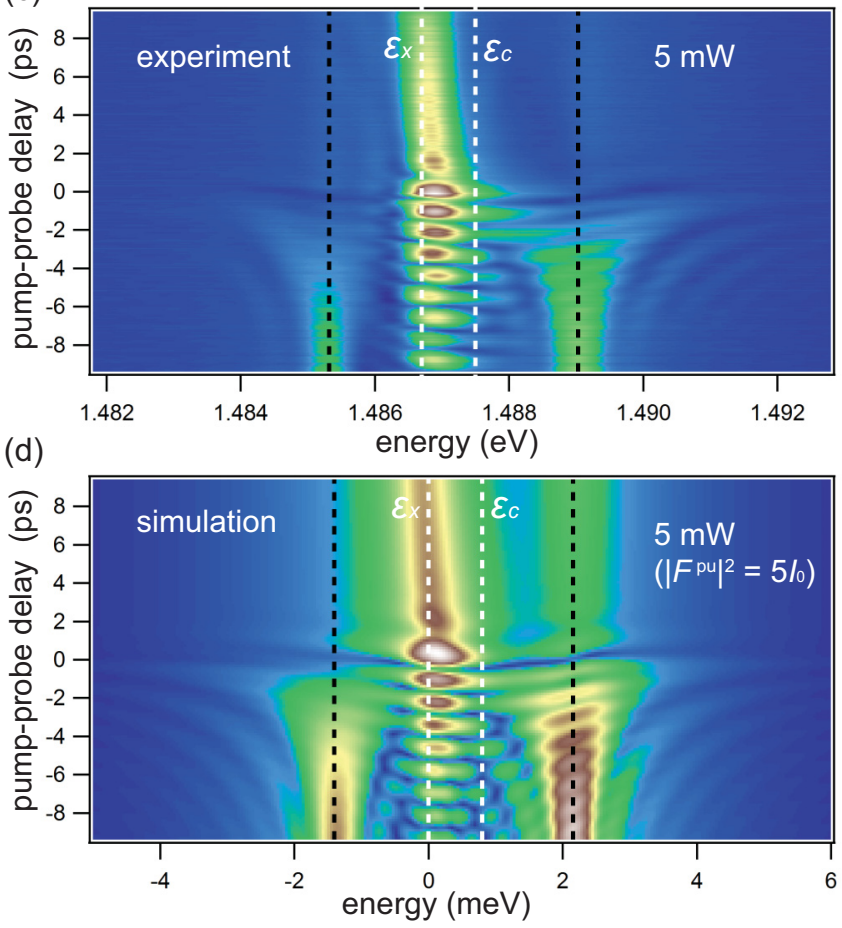

FIG. 1. (Color online) Measured and simulated probe transmission are shown as a function of energy and time delay between pump and probe pulse. The spectra are measured for two different pump intensities: (a) $1.48 \times 10^{13}(1 \mathrm{~mW})$ and (c) $7.4 \times 10^{13}(5 \mathrm{~mW})$ photons pulse $\mathrm{cm}^{-1} \mathrm{~cm}^{-2}$. Corresponding simulated spectra are attached below the measured spectra [(b) and (d)]. The black dashed lines represent the lower- and upper-polariton peak energies without pump pulse. The white dashed lies are the cavity photon and exciton energies. In the simulation, the intensity $I_{0}$ is defined as $I_{0}=0.8 / g_{0}$. upper) at both positive and negative delays and the lower polariton shows a maximum blue shift at zero delay. The delay dependence of the lower-polariton blue shift is asymmetric with respect to zero delay. While the blue shift gradually decreases at negative delays, it stays for a long time at positive delays. No clear energy shift of the upper-polariton resonance is seen for all delays. At high pump intensity [Fig. 1(c)], a triple-peak structure appears at negative delays, while a single peak exists at positive delays. With the aid of numerical simulations based on EBE, we show that the observed behavior originates from a long-living incoherent population and a short-living coherent polarization of excitons.

\section{THEORY}

For the analysis of the experiment, we use EBE [19,20]. The starting point of EBE is a bosonic exciton-photon Hamiltonian:

$$
\begin{aligned}
\hat{H}= & \int d \mathbf{x}\left[\epsilon_{x} \hat{\boldsymbol{\psi}}_{x}^{\dagger} \hat{\boldsymbol{\psi}}_{x}+\hat{\boldsymbol{\psi}}_{c}^{\dagger}\left(\epsilon_{c}-\frac{\hbar^{2} \nabla^{2}}{2 m_{c}}\right) \hat{\boldsymbol{\psi}}_{c}\right. \\
& \left.+\Omega\left(\hat{\boldsymbol{\psi}}_{c}^{\dagger} \hat{\boldsymbol{\psi}}_{x}+\hat{\boldsymbol{\psi}}_{x}^{\dagger} \hat{\boldsymbol{\psi}}_{c}\right)\right] \\
& +\int d \mathbf{x} \int d \mathbf{x}^{\prime}\left[\frac{1}{2} \hat{\boldsymbol{\psi}}_{x}^{\dagger} \hat{\boldsymbol{\psi}}_{x}^{\dagger \prime} V_{\mathrm{ex}}\left(\mathbf{x}-\mathbf{x}^{\prime}\right) \hat{\boldsymbol{\psi}}_{x} \hat{\boldsymbol{\psi}}_{x}^{\prime}\right. \\
& \left.-\frac{1}{2} V_{\mathrm{pae}}\left(\mathbf{x}-\mathbf{x}^{\prime}\right)\left(\hat{\boldsymbol{\psi}}_{c}^{\dagger} \hat{\boldsymbol{\psi}}_{x}^{\dagger \prime} \hat{\boldsymbol{\psi}}_{x} \hat{\boldsymbol{\psi}}_{x}^{\prime}+\hat{\boldsymbol{\psi}}_{x}^{\dagger} \hat{\boldsymbol{\psi}}_{x}^{\dagger \prime} \hat{\boldsymbol{\psi}}_{x} \hat{\boldsymbol{\psi}}_{c}^{\prime}\right)\right]
\end{aligned}
$$

$\hat{\boldsymbol{\psi}}_{x(c)}$ and $\hat{\boldsymbol{\psi}}_{x(c)}^{\dagger}$ are exciton (photon) field creation and annihilation operators. They obey the boson commutation relations $\left[\hat{\boldsymbol{\psi}}_{x(c)}, \hat{\boldsymbol{\psi}}_{x(c)}^{\dagger \prime}\right]=\delta\left(\mathbf{x}-\mathbf{x}^{\prime}\right)$ and $\left[\hat{\boldsymbol{\psi}}_{x(c)}, \hat{\boldsymbol{\psi}}_{x(c)}^{\prime}\right]=0$. This Hamiltonian can be obtained from the electron-hole Hamiltonian via a boson mapping method called the Usui transformation [19]. Since the exciton mass is large, the kinetic term of the exciton is neglected. The interactions are assumed to be contact interactions: $V_{\mathrm{ex}}\left(\mathbf{x}-\mathbf{x}^{\prime}\right)=g \delta\left(\mathbf{x}-\mathbf{x}^{\prime}\right)$ and $V_{\text {pae }}(\mathbf{x}-$ $\left.\mathbf{x}^{\prime}\right)=2 g_{\text {pae }} \delta\left(\mathbf{x}-\mathbf{x}^{\prime}\right)[22,23]$. The exciton-exciton interaction potential $V_{\mathrm{ex}}$ is associated with the Coulomb exchange scattering. The term $V_{\text {pae }}$ is a photon-assisted exchange scattering [24] and contributes to the reduction of the Rabi coupling, which is the reminiscence of the fermionic nature of the exciton [19]. In order to obtain a closed set of equations, we truncate the hierarchy by applying the following assumptions such as $\left\langle\hat{\boldsymbol{\psi}}_{x}^{\dagger} \hat{\boldsymbol{\psi}}_{x} \hat{\boldsymbol{\psi}}_{x}\right\rangle \simeq\left\langle\hat{\boldsymbol{\psi}}_{x}^{\dagger} \hat{\boldsymbol{\psi}}_{x}\right\rangle\left\langle\hat{\boldsymbol{\psi}}_{x}\right\rangle,\left\langle\hat{\boldsymbol{\psi}}_{x}^{\dagger} \hat{\boldsymbol{\psi}}_{x} \hat{\boldsymbol{\psi}}_{c}\right\rangle \simeq$ $\left\langle\hat{\boldsymbol{\psi}}_{x}^{\dagger} \hat{\boldsymbol{\psi}}_{x}\right\rangle\left\langle\hat{\boldsymbol{\psi}}_{c}\right\rangle,\left\langle\hat{\boldsymbol{\psi}}_{c}^{\dagger} \hat{\boldsymbol{\psi}}_{x} \hat{\boldsymbol{\psi}}_{x}\right\rangle \simeq\left\langle\hat{\boldsymbol{\psi}}_{c}^{\dagger}\right\rangle\left\langle\hat{\boldsymbol{\psi}}_{x} \hat{\boldsymbol{\psi}}_{x}\right\rangle$, and $\left\langle\hat{\boldsymbol{\psi}}_{x} \hat{\boldsymbol{\psi}}_{x}\right\rangle=0$. We define the exciton population as $N(\mathbf{x}, t)=\left\langle\hat{\boldsymbol{\psi}}_{x}^{\dagger} \hat{\boldsymbol{\psi}}_{x}\right\rangle$ and the exciton polarization as $P(\mathbf{x}, t)=\left\langle\hat{\boldsymbol{\psi}}_{x}\right\rangle$. Assuming factorization between the photon and exciton, we define $E(\mathbf{x}, t)=\left\langle\hat{\psi}_{c}\right\rangle$. With the aid of the Heisenberg equation of motion, the EBE then reads [25]

$$
\begin{aligned}
i \hbar \dot{N} & =-i \Gamma_{x} N-2 i\left(\Omega-2 g_{\mathrm{pae}} N\right) \operatorname{Im}\left[P E^{*}\right] \\
i \hbar \dot{P} & =\left(\epsilon_{x}+g_{0} N-i \gamma_{x}(N)\right) P+\left(\Omega-2 g_{\mathrm{pae}} N\right) E \\
i \hbar \dot{E} & =\left(\epsilon_{c}-\frac{\hbar^{2}}{2 m_{c}} \nabla^{2}-i \gamma_{c}\right) E+\left(\Omega-g_{\mathrm{pae}} N\right) P-f_{\mathrm{ext}} .
\end{aligned}
$$

To obtain the above equations, the interaction constant $g$ is phenomenologically divided into a real and imaginary part: 
$g=g_{0}-i g^{\prime}$. The real part $g_{0}$ is associated with an energy renormalization, while the imaginary part $g^{\prime}$ represents the strength of EID, which is also referred to as collisional broadening. The ratio of the constants is estimated as $2 g_{\text {pae }} / g_{0} \simeq$ $\hbar \Omega / 6 n_{s} E_{b} a_{0}^{2}$, where $n_{s}$ is the saturation density of excitons [26]. $E_{b}$ and $a_{0}$ are respectively the exciton binding energy and Bohr radius. The constants, $\gamma_{x}(N)$ and $\Gamma_{x}$ are respectively the polarization dephasing and the population decay rate of excitons. In general, $\gamma_{x}(N)$ is written as [14,27,28],

$$
\gamma_{x}(N)=\Gamma_{x} / 2+\gamma_{x}^{*}+g^{\prime} N,
$$

where $\gamma_{x}^{*}$ is the pure dephasing term. In the terminology of two level systems, $\Gamma_{x}$ and $\gamma_{x}$ correspond to the inverse of $T_{1}$ and $T_{2}$ times respectively. The EID constant $g^{\prime}$ introduces a phenomenological linear increase of the dephasing that depends on the exciton population $N$, which plays an important role in our experiment.

The advantage of EBE, compared to GPE, is that we can apply independent decay rates for the coherent polarization and incoherent population and calculate the time evolution of each. Indeed, in the commonly used GPE, a factorization, $\left\langle\hat{\boldsymbol{\psi}}_{x}^{\dagger} \hat{\boldsymbol{\psi}}_{x}\right\rangle=\left\langle\hat{\boldsymbol{\psi}}_{x}^{\dagger}\right\rangle\left\langle\hat{\boldsymbol{\psi}}_{x}\right\rangle$ is implicitly assumed [23]. Therefore, the dynamics of the population $N(\mathbf{x}, t)$ is uniquely determined by the polarization $P(\mathbf{x}, t)$ through $N=|P|^{2}$. In the EBE, this condition is satisfied when neither pure dephasing nor EID exist: $\gamma_{x}^{*}=0$ and $g^{\prime}=0$ (coherent limit). The EBE are a close analog of the optical Bloch equations (OBE) [16,29], however, differing since OBE are based on a two-level electron-hole system, while EBE are based on a bosonic exciton basis [19].

To reproduce the experiments, $\Gamma_{x}$ and $\gamma_{c}$ are chosen to be $0.01 \mathrm{meV}$ and $0.1 \mathrm{meV}$ respectively. The pure dephasing is set to $\gamma_{x}^{*}=0.1 \mathrm{meV}$ [30], additionally, we include EID as the primary decoherence mechanism in our simulations. We set the interaction constants as $g^{\prime}=0.4 g_{0}$ and $g_{\text {pae }}=0.3 g_{0} . f_{\text {ext }}$ is the excitation photon field and is assumed to be a Gaussian pulse. The energy of both pump and probe pulses is set at the center of the lower and upper polariton branches.

For the calculation of the pump-probe dynamics, we use a coupled-mode approximation: $N(\mathbf{x}, t)=N^{p u}+N^{p r} e^{i \boldsymbol{k} \cdot \mathbf{x}}+$ $N^{p r *} e^{-i \boldsymbol{k} \cdot \mathbf{x}}$ (the population is a real value), $P(\mathbf{x}, t)=$ $P^{p u}+P^{p r} e^{i \boldsymbol{k} \cdot \mathbf{x}}+P^{i d} e^{-i \boldsymbol{k} \cdot \mathbf{x}}$, and $E(\mathbf{x}, t)=E^{p u}+E^{p r} e^{i \boldsymbol{k} \cdot \mathbf{x}}+$ $E^{i d} e^{-i \boldsymbol{k} \cdot \mathbf{x}}$. For example, $P^{p u}, P^{p r}$, and $P^{i d}$ represent the pump, probe, and idler component of the polarization, respectively. Considering the conservation of momentum, we obtain eight coupled equations. The detail of the coupled-mode equations is explained in the Appendix. The pump and probe pulses are introduced as $E^{p u}$ and $E^{p r}$ respectively and the transmitted probe signal is obtained through $E^{p r}$. This is the standard method of calculating a transient four-wave mixing signal in optical Bloch equations [31,32]. Since the wave number of the probe is sufficiently small, we neglect the momentum dispersion of the photon mode.

\section{RESULTS}

\section{A. Incoherent regime}

The simulated probe transmission spectra are given in Figs. 1(b) and 1(d) for two different pump intensities. There are striking similarities between the experimental and simulated (a) time evolution of probe $\left|E^{\text {pr }}(t)\right|$ at $\tau=3.5 \mathrm{ps}$

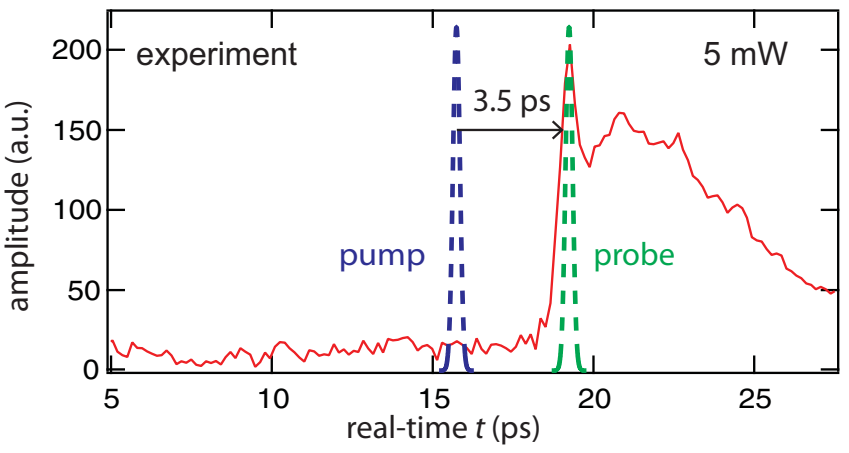

(b) time evolution of probe $\left|E^{p r}(t)\right|$ at $\tau=-3.5 \mathrm{ps}$

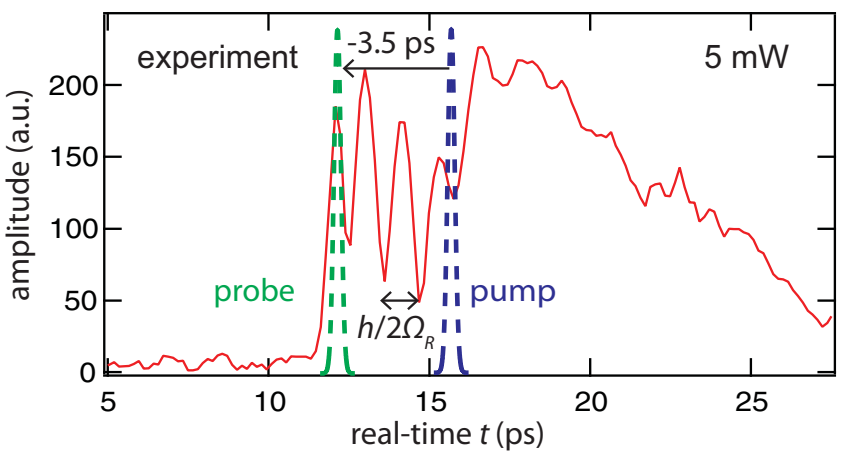

FIG. 2. (Color online) Realtime evolution of the probe signal from the experiment shown in Fig. 1 at two selected pump probe delay times (a) $\tau=+3.5$ ps and (b) $\tau=-3.5$ ps. For $\tau=+3.5$ ps a single region, corresponding to the central peak, is present. For $\tau=-3.5 \mathrm{ps}$, two distinct regions are observed: an oscillating signal is present prior to the arrival of the pump corresponding to the two polariton modes and the nonoscillating signal after the pump arrives, which corresponds to the central peak.

spectra. First, Fig. 1(b) features a long-lasting $\left(\sim \hbar / \Gamma_{x}\right)$ blue shift of the lower polariton in the positive delay, while the blue shift builds up on a shorter time scale $\left[\sim 2 \hbar /\left(\gamma_{c}+\gamma_{x}^{*}\right)\right]$ in the negative delay. On the other hand, the energy shift of the upper polariton is almost zero because of the cancellation of the blue and red shift contributions originating from the terms $g_{0}$ and $g_{\text {pae }}$, respectively. The high-density simulation [Fig. 1(d)] reproduces both the occurrence of three peaks at negative delays and of a single peak at positive delays. At negative delays, the three-peak structure appears because the signal is integrated over time during the duration of the probe pulse in the sample. To elucidate this point, the realtime evolution of the probe pulse for both negative and positive pump-probe delays is shown in Fig. 2. The probe signal at negative pump-probe delays [e.g., Fig. 2(b)] consists of two distinct regions: before and after the arrival of the pump. When spectrally resolved, the region prior to the pump arrival gives the two polariton modes, which oscillate with a period $h / 2 \Omega_{R}$, this is a direct manifestation of the Rabi oscillation between an exciton and a photon. After the arrival of the pump, the remaining coherent probe population undergoes a strong energy shift, approaching the cavity mode, which results in a single central peak. For positive pump-probe delays [e.g., Fig. 2(a)], the entire probe evolution occurs in the presence 

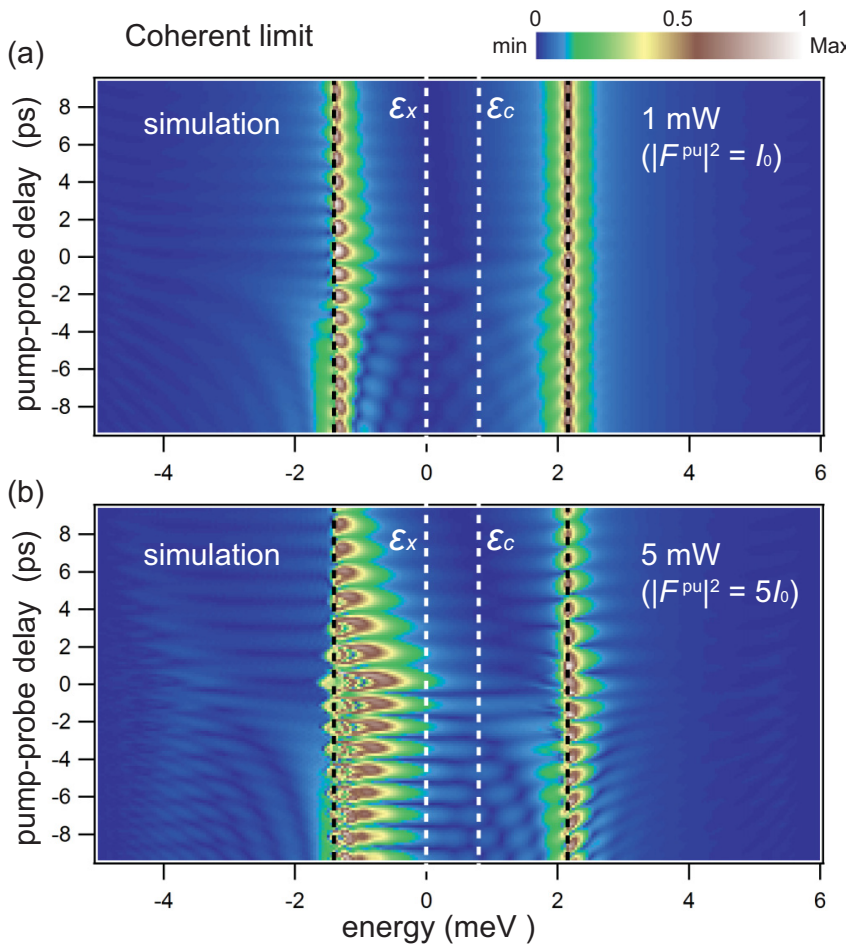

FIG. 3. (Color online) Simulated probe transmission as a function of energy and pump-probe time delay without EID or pure dephasing $\left(g^{\prime}=\gamma_{x}^{*}=0 \mathrm{meV}\right)$. The other parameters are the same as those used in the simulation of Figs. 1(a) and 1(b) respectively correspond to $1 \mathrm{~mW}$ and $5 \mathrm{~mW}$ pump intensities.

of the pump population and therefore only the central peak is observed. This is a similar mechanism to the one operating for the dynamical blue shift predicted by the GPE [see Fig. 3(b)]. If the pump intensity is further increased, eventually the term $\gamma_{x}(N)$ becomes comparable to the effective Rabi coupling $\Omega-2 g_{\text {gpae }} N$ and the central peak asymptotically reaches the cavity mode $\epsilon_{c}$ (see Fig. 4), which is the signature of a strong to weak coupling transition $[33,34]$.

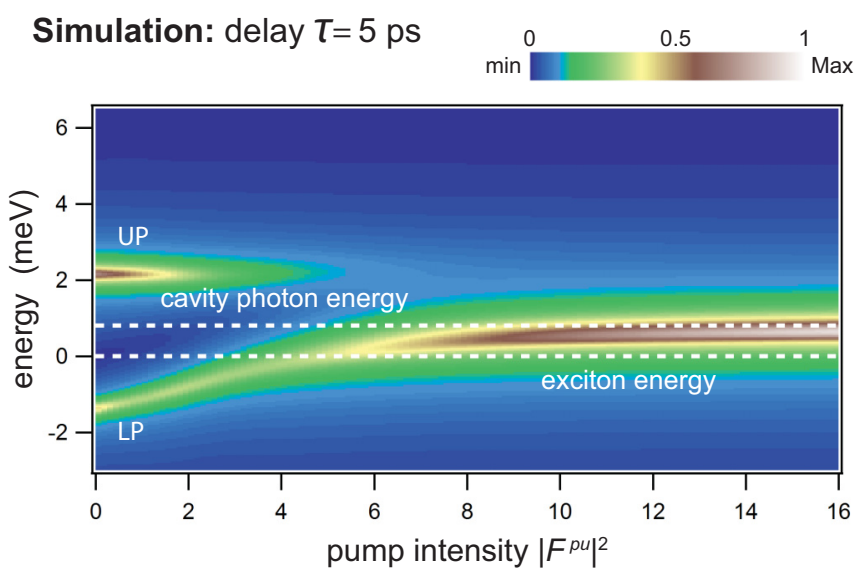

FIG. 4. (Color online) Simulated probe transmission at a pumpprobe delay of $5 \mathrm{ps}$. The increasing pump power drives the system into the weak coupling regime $\left(\epsilon_{L P}=\epsilon_{c}\right)$ asymptotically.

\section{B. Coherent regime}

In order to further elucidate the effect of the incoherent exciton population and of EID on the two polariton resonances, we present in Fig. 3 a simulation without EID or pure dephasing $\left(g^{\prime}=\gamma_{x}^{*}=0\right)$. The other parameters are same as for Fig. 1. The probe transmission of Fig. 3 is a simulation of the coherent limit, where the polarization decay (dephasing) rate is a half of the population decay rate $\left[\gamma_{x}(N)=\Gamma_{x} / 2=0.005 \mathrm{meV}\right]$. We find that the polariton branch is broadened towards the high energy side because of dynamical energy shift. Namely, the mean-field energy shift of polaritons temporarily decreases following the decay of the polariton density. The time integration of the temporal decrease of the energy shift introduces a broadening of the polariton branches. The blue shift of both polariton resonances decays twice faster at positive delays than they emerge at negative ones. In this limit, the dynamics of the exciton population is uniquely determined by the polarization and we can replace the population $N(\mathbf{x}, t)$ with the square of the polarization $|P(\mathbf{x}, t)|^{2}$. Here the three sets of equations can be reduced to two equations composed of the exciton polarization and electric field, this is the commonly used exciton-photon GPE [23,35]. Clearly, the GPE cannot reproduce the dynamics of polaritons in the presence of EID, which is evidenced by the huge differences between Fig. 3 and the experiments [Figs. 1(a) and 1(c)].

In particular, the high pump intensity simulation [Fig. 3(b)] reproduces neither the three peak structure nor the disappearance of the quantum beat pattern for the positive delay. From this simulation we infer that the bleaching of the upper and lower polariton resonances at positive delays [Fig. 1(c)] is associated with EID. The transition to the weak coupling regime can also be driven with the GPEs (not shown), however the very strong dynamical blue shift effects completely differ from the observed experimental behavior.

\section{Comparison of coherent and incoherent regimes}

In Fig. 5, we present simulated real-time evolutions of exciton polarization $P(t)$ and population $N(t)$ at $\boldsymbol{k}=0 \mu \mathrm{m}^{-1}$ after the arrival of a single laser pulse. Without EID (coherent limit), the time evolution of the exciton population $N(t)$ is found to coincide with that of $|P(t)|^{2}$, which supports the relation $N(t)=|P(t)|^{2}$ and the factorization $\left\langle\hat{\psi}_{x}^{\dagger} \hat{\boldsymbol{\psi}}_{x}\right\rangle=$ $\left\langle\hat{\boldsymbol{\psi}}_{x}^{\dagger}\right\rangle\left\langle\hat{\boldsymbol{\psi}}_{x}\right\rangle$, which is assumed in the exciton-photon GPE. In this case, the lifetime of the system is mainly determined by the short photon lifetime $\left(\sim \hbar / \gamma_{c}\right)$. On the other hand, with EID, we have to distinguish between the dynamics of the polarization and that of the population. While the exciton polarization $P(t)$ is directly coupled to the cavity photon field $E(t)$ (not shown), there is no direct coupling between the exciton population $N(t)$ and the photon $E(t)$. Therefore, while the polarization decays with a lifetime of the same order as that of the cavity photon $\left(\sim \hbar / \gamma_{c}\right)$, the population decays independently and stays for a long time $\left(\sim \hbar / \Gamma_{x}\right)$, even after the disappearance of the polarization. Intuitively, the EID process can be understood as follows; microscopically, the exciton-exciton collisions introduce an energy fluctuation, which gives an additional random phase to the time evolution of the exciton field operator $\hat{\psi}_{x}(\mathbf{x}, 0) e^{i\left(\epsilon_{x}+\delta \epsilon\right) t / \hbar}$. Since the 

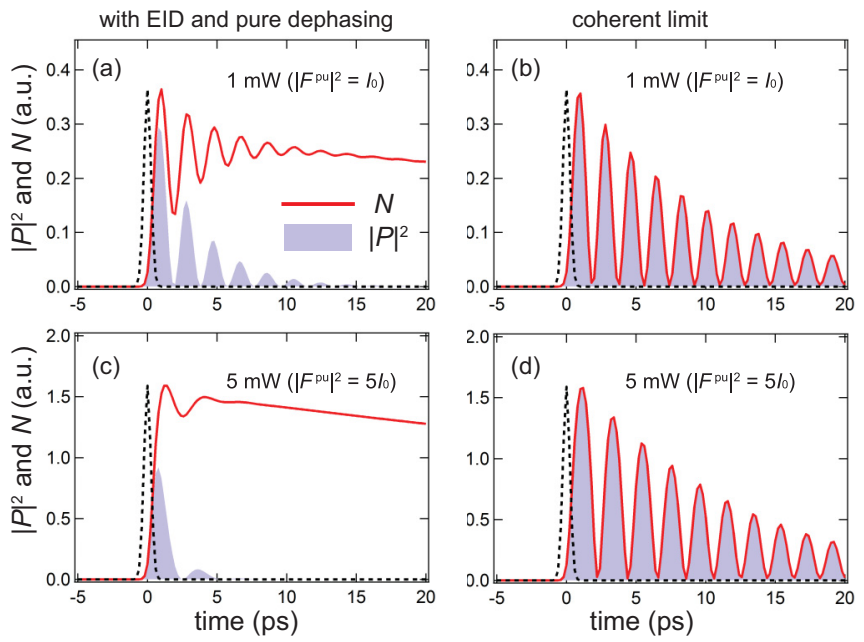

FIG. 5. (Color online) Simulated time evolution of the $\boldsymbol{k}=$ $0 \mu \mathrm{m}^{-1}$ polarization $|P|^{2}$ and population $N$ as a function of time after an arrival of a single pulse. Simulations with (a), (c) EID and pure dephasing and in (b), (d) a coherent limit $\left(g^{\prime}=\gamma_{x}^{*}=0 \mathrm{meV}\right)$ are presented for (a), (b) two different laser pulse intensities $1 \mathrm{~mW}$ and (c), (d) $5 \mathrm{~mW}$. The parameters are the same as the simulation of Fig. 1. The dashed lines represent scaled laser pulses.

phase $\delta \epsilon$ is random, the expectation value $P(\mathbf{x}, t)=\left\langle\hat{\boldsymbol{\psi}}_{x}(\mathbf{x}, t)\right\rangle$ shows a time decay [36], which is the origin of the imaginary part of the interaction constant $g^{\prime}$ (EID) [28,37]. Meanwhile, the energy fluctuations affect neither the term $\hat{\psi}_{x}^{\dagger}(\mathbf{x}, t) \hat{\boldsymbol{\psi}}_{x}(\mathbf{x}, t)$ nor its expectation value $N(\mathbf{x}, t)=\left\langle\hat{\boldsymbol{\psi}}_{x}^{\dagger}(\mathbf{x}, t) \hat{\boldsymbol{\psi}}_{x}(\mathbf{x}, t)\right\rangle$ due to a phase cancellation [36]. Finally, we comment that the incoherent exciton population $N$ should be interpreted as the inactive excitonic reservoir already discussed in the context of nonresonantly excited polariton condensates [38,39]. Actually, both the incoherent exciton population, featured in Fig. 5, and the inactive excitonic reservoir have long lifetimes and substantially contribute to the energy shift of the polariton resonances [40].

\section{CONCLUSION}

In conclusion, we have studied the coherent dynamics of exciton-polaritons by femtosecond pump-probe spectroscopy in a high-quality semiconductor microcavity. In this investigation we have considered explicitly the different mechanisms for polariton-polariton interactions: the exciton-exciton and the photon-assisted exchange scattering. On the theoretical side, calculation of the EID term has only been attempted for a coherently excited population of lower polaritons [41]. In view of our results, further estimation of this collisional scattering rate should include the contribution of both excitonexciton Coulomb scattering and photon-assisted exchange scattering between polaritons. Both the experimental and the theoretical results demonstrate that dephasing effects mediated by exciton-exciton interactions are a major mechanism for the breakdown of the strong coupling regime and for establishing a long-lived exciton population. This is evidenced by a power-dependent long-lived strong perturbation on the experimental pump-probe signal, which is successfully simulated with the inclusion of EID in the excitonic Bloch equations. Consequently, we establish that simulation of the polariton dynamics based on the excitonic Bloch equations provides a complete description of the experimental results, which cannot be replicated by the standard Gross-Pitaevskii equations.

\section{ACKNOWLEDGMENTS}

The present work is supported by the Swiss National Science Foundation under Project No. 153620 and the European Research Council under project Polaritonics Contract No. 291120. The polatom network is also acknowledged.

\section{APPENDIX: COUPLED-MODE EQUATIONS}

In this Appendix, we present the explicit forms of the coupled-mode equations [31,32] used to simulate the pumpprobe spectra. We restrict the number of modes to the three main ones: pump, probe, and idler. The polarization $P(\mathbf{x}, t)$, population $N(\mathbf{x}, t)$ and electric field $E(\mathbf{x}, t)$ are respectively written as $N(\mathbf{x}, t)=N^{p u}+N^{p r} e^{i \boldsymbol{k} \cdot \mathbf{x}}+N^{p r *} e^{-i \boldsymbol{k} \cdot \mathbf{x}}, P(\mathbf{x}, t)=$ $P^{p u}+P^{p r} e^{i \boldsymbol{k} \cdot \mathbf{x}}+P^{i d} e^{-i \boldsymbol{k} \cdot \mathbf{x}}$, and $E(\mathbf{x}, t)=E^{p u}+E^{p r} e^{i k \cdot \mathbf{x}}+$ $E^{i d} e^{-i \boldsymbol{k} \cdot \mathbf{x}}$. Substituting these representations into the excitonic Bloch equations given in the manuscript and neglecting components such as $e^{ \pm i 2 k}$ and $e^{ \pm i 3 k}[31,32]$, we obtain eight coupled equations of motion. First, the equations for the population $N$ reads,

$$
\begin{aligned}
i \hbar \dot{N}^{p u}= & -i \Gamma_{x} N^{p u}-\Omega a_{p u} \\
& +2 g_{\text {pae }}\left(a_{p u} N^{p u}+b_{i d} N^{p r}+b_{p r} N^{p r *}\right) \\
i \hbar \dot{N}^{p r}= & -i \Gamma_{x} N^{p r}-\Omega b_{p r} \\
& +2 g_{\text {pae }}\left(b_{p r} N^{p u}+a_{p u} N^{p r}+c N^{p r *}\right) .
\end{aligned}
$$

In a similar way, the equations of motion of exciton polarizations are written as

$$
\begin{aligned}
i \hbar \dot{P}^{p u}= & \left(\epsilon_{x}-i \gamma_{x}\right) P^{p u} \\
+ & g\left(N^{p u} P^{p u}+N^{p r *} P^{p r}+N^{p r} P^{i d}\right)+\Omega E^{p u} \\
- & 2 g_{\mathrm{pae}}\left(N^{p u} E^{p u}+N^{p r *} E^{p r}+N^{p r} E^{i d}\right) \\
i \hbar \dot{P}^{p r}= & \left(\epsilon_{x}-i \gamma_{x}\right) P^{p r}+g\left(N^{p u} P^{p r}+N^{p r} P^{p u}\right) \\
& +\Omega E^{p r}-2 g_{\mathrm{pae}}\left(N^{p u} E^{p r}+N^{p r} E^{p u}\right) \\
i \hbar \dot{P}^{i d}= & \left(\epsilon_{x}-i \gamma_{x}\right) P^{i d}+g\left(N^{p u} P^{i d}+N^{p r *} P^{p u}\right) \\
& +\Omega E^{i d}-2 g_{\mathrm{pae}}\left(N^{p u} E^{i d}+N^{p r *} E^{p u}\right) .
\end{aligned}
$$

Finally, the electric fields follow the following equations:

$$
\begin{aligned}
i \hbar \dot{E}^{p u}= & \left(\epsilon_{c}-i \gamma_{c}\right) E^{p u}+\Omega P^{p u} \\
- & g_{\mathrm{pae}}\left(N^{p u} P^{p u}+N^{p r} P^{i d}+N^{p r *} P^{p r}\right) \\
- & f_{\mathrm{ext}}^{p u} \\
i \hbar \dot{E}^{p r}= & \left(\epsilon_{c}-i \gamma_{c}\right) E^{p r}+\Omega P^{p r} \\
& -g_{\mathrm{pae}}\left(N^{p u} P^{p r}+N^{p r} P^{p u}\right)-f_{\mathrm{ext}}^{p r} \\
i \hbar \dot{E}^{i d}= & \left(\epsilon_{c}-i \gamma_{c}\right) E^{i d}+\Omega P^{i d} \\
& -g_{\mathrm{pae}}\left(N^{p u} P^{i d}+N^{p r *} P^{p u}\right) .
\end{aligned}
$$


In the equations of populations [Eq. (A1) and (A2)], the quantities $a_{p u}, b_{p r}, b_{i d}$, and $c$ are respectively given by

$$
\begin{aligned}
a_{p u} & =2 i \operatorname{Im}\left(P^{p u} E^{p u *}+P^{p r} E^{p r *}+P^{i d} E^{i d *}\right) \\
b_{p r} & =P^{p u} E^{i d *}-P^{i d *} E^{p u}+P^{p r} E^{p u *}-P^{p u *} E^{p r} \\
b_{i d} & =P^{p u} E^{p r *}-P^{p r *} E^{p u}+P^{i d} E^{p u *}-P^{p u *} E^{i d} \\
c & =P^{p r} E^{i d *}-P^{i d *} E^{p r} .
\end{aligned}
$$

In Eqs. (A6) and (A7), $f_{\mathrm{ext}}^{p u(p r)}$ respectively the pump (probe) excitation photon field is taken as a Gaussian pulse

$$
\begin{aligned}
f_{\mathrm{ext}}^{p u(p r)}= & F^{p u(p r)} \exp \left(-\frac{\left(t-t_{p u(p r)}\right)^{2}}{\tau^{2}}\right) \\
& \times \exp \left[-i\left(\epsilon_{0}^{\mathrm{pulse}} / \hbar\right)\left(t-t_{p u(p r)}\right)\right] .
\end{aligned}
$$

We set the energy of the pulses $\epsilon_{0}^{\text {pulse }}$ at the center of both polariton branches $\left(\epsilon_{0}^{\text {pulse }}=\epsilon_{x} / 2+\epsilon_{c} / 2\right)$ and set the pulse duration $\tau$ to 0.5 ps. $t_{p u(p r)}$ is defined as the incident time of the pump (probe) pulse.
[1] C. Weisbuch, M. Nishioka, A. Ishikawa, and Y. Arakawa, Phys. Rev. Lett. 69, 3314 (1992).

[2] J. Kasprzak, M. Richard, S. Kundermann, A. Baas, P. Jeambrun, J. Keeling, F. Marchetti, M. H. Szymanska, R. André, J. L. Staehli, V. Savona, P. B. Littlewood, B. Deveaud, and L. S. Dang, Nature (London) 443, 409 (2006).

[3] H. Deng, G. Weihs, C. Santori, J. Bloch, and Y. Yamamoto, Science 298, 199 (2002).

[4] A. Amo, J. Lefrère, S. Pigeon, C. Adrados, C. Ciuti, I. Carusotto, R. Houdré, E. Giacobino, and A. Bramati, Nat. Phys. 5, 805 (2009).

[5] V. Kohnle, Y. Léger, M. Wouters, M. Richard, M. T. PortellaOberli, and B. Deveaud-Plédran, Phys. Rev. Lett. 106, 255302 (2011).

[6] V. Kohnle, Y. Léger, M. Wouters, M. Richard, M. T. PortellaOberli, and B. Deveaud, Phys. Rev. B 86, 064508 (2012).

[7] A. Huynh, J. Tignon, Ph. Roussignol, C. Delalande, R. André, R. Romestain, and D. Le Si Dang, Phys. Rev. B 66, 113301 (2002).

[8] A. Huynh, J. Tignon, G. Keller, P. Roussignol, C. Delalande, R. André, R. Romestain, and L. S. Dang, Phys. Rev. B 68, 165340 (2003).

[9] N. Takemura, S. Trebaol, M. D. Anderson, V. Kohnle, Y. Léger, D. Y. Oberli, M. T. Portella-Oberli, and B. Deveaud, Phys. Rev. B 92, 125415 (2015).

[10] M. Vladimirova, S. Cronenberger, D. Scalbert, K. V. Kavokin, A. Miard, A. Lemaître, J. Bloch, D. Solnyshkov, G. Malpuech, and A. V. Kavokin, Phys. Rev. B 82, 075301 (2010).

[11] D. Scalbert, M. Vladimirova, A. Brunetti, S. Cronenberger, M. Nawrocki, J. Bloch, A. Kavokin, I. Shelykh, R. André, D. Solnyshkov, and G. Malpuech, Superlattices Microstruct. 43, 417 (2008), Proceedings of the 7th International Conference on Physics of Light-Matter Coupling in Nanostructures.

[12] N. Takemura, S. Trebaol, M. Wouters, M. T. Portella-Oberli, and B. Deveaud, Phys. Rev. B 90, 195307 (2014).

[13] N. Takemura, S. Trebaol, M. Wouters, M. T. Portella-Oberli, and B. Deveaud, Nat. Phys. 10, 500 (2014).

[14] J. Shah, Ultrafast Spectroscopy of Semiconductors and Semiconductor Nanostructures, Vol. 115 (Springer Science \& Business Media, Berlin, 1999).

[15] M. Kira and S. Koch, Prog. Quant. Electron. 30, 155 (2006).

[16] H. Wang, H. Q. Hou, and B. E. Hammons, Phys. Rev. Lett. 81, 3255 (1998).
[17] M. Wouters and I. Carusotto, Phys. Rev. Lett. 99, 140402 (2007).

[18] M. Wouters, T. K. Paraiso, Y. Léger, R. Cerna, F. MorierGenoud, M. T. Portella-Oberli, and B. Deveaud-Plédran, Phys. Rev. B 87, 045303 (2013).

[19] G. Rochat, C. Ciuti, V. Savona, C. Piermarocchi, A. Quattropani, and P. Schwendimann, Phys. Rev. B 61, 13856 (2000).

[20] D. Wang and M. M. Dignam, Phys. Rev. B 79, 165320 (2009).

[21] R. Stanley, R. Houdre, U. Oesterle, M. Gailhanou, and M. Ilegems, Appl. Phys. Lett. 65, 1883 (1994).

[22] L. Pitaevskii and S. Stringari, Bose-Einstein Condensation, International Series of Monographs on Physics (Clarendon, Oxford, 2003).

[23] I. Carusotto and C. Ciuti, Rev. Mod. Phys. 85, 299 (2013).

[24] M. Combescot, M. Dupertuis, and O. Betbeder-Matibet, Europhys. Lett. 79, 17001 (2007).

[25] Note that Rochat's equation for the polarization (Ref. [19]) differ by a factor of 2 in the energy shift term.

[26] D. Sarchi and V. Savona, Phys. Rev. B 75, 115326 (2007).

[27] T. Baars, M. Bayer, A. Forchel, F. Schäfer, and J. P. Reithmaier, Phys. Rev. B 61, R2409(R) (2000).

[28] C. Ciuti, V. Savona, C. Piermarocchi, A. Quattropani, and P. Schwendimann, Phys. Rev. B 58, 7926 (1998).

[29] X. Li, T. Zhang, C. N. Borca, and S. T. Cundiff, Phys. Rev. Lett. 96, 057406 (2006).

[30] A. Honold, L. Schultheis, J. Kuhl, and C. W. Tu, Phys. Rev. B 40, 6442 (1989).

[31] T. Yajima and Y. Taira, J. Phys. Soc. Jpn. 47, 1620 (1979).

[32] D. B. Turner, P. Wen, D. H. Arias, and K. A. Nelson, Phys. Rev. B 84, 165321 (2011).

[33] H. Cao, S. Pau, J. M. Jacobson, G. Björk, Y. Yamamoto, and A. Imamoğlu, Phys. Rev. A 55, 4632 (1997).

[34] V. Savona, L. Andreani, P. Schwendimann, and A. Quattropani, Solid State Commun. 93, 733 (1995).

[35] Due to the coupled-mode approximation, the simulation results of the EBE in the coherent limit are slightly different from those of coupled-mode GPE. This is because terms such as $N^{2 p r} e^{-i 2 \boldsymbol{k} \cdot \mathbf{x}}$ and $N^{2 p r *} e^{-i 2 \boldsymbol{k} \cdot \mathbf{x}}$ are neglected in this approximation.

[36] S. Schmitt-Rink, S. Mukamel, K. Leo, J. Shah, and D. S. Chemla, Phys. Rev. A 44, 2124 (1991).

[37] C. Cohen-Tannoudji, J. Dupont-Roc, G. Grynberg, and P. Thickstun, Atom-Photon Interactions: Basic Processes and Applications (Wiley Online Library, New York, 1992). 
[38] K. G. Lagoudakis, F. Manni, B. Pietka, M. Wouters, T. C. H. Liew, V. Savona, A. V. Kavokin, R. André, and B. DeveaudPlédran, Phys. Rev. Lett. 106, 115301 (2011).

[39] M. De Giorgi, D. Ballarini, P. Cazzato, G. Deligeorgis, S. I. Tsintzos, Z. Hatzopoulos, P. G. Savvidis, G. Gigli, F. P. Laussy, and D. Sanvitto, Phys. Rev. Lett. 112, 113602 (2014).
[40] L. Ferrier, E. Wertz, R. Johne, D. D. Solnyshkov, P. Senellart, I. Sagnes, A. Lemaitre, G. Malpuech, and J. Bloch, Phys. Rev. Lett. 106, 126401 (2011).

[41] C. Ciuti, V. Savona, C. Piermarocchi, A. Quattropani, and P. Schwendimann, Phys. Rev. B 58, R10123 (1998). 